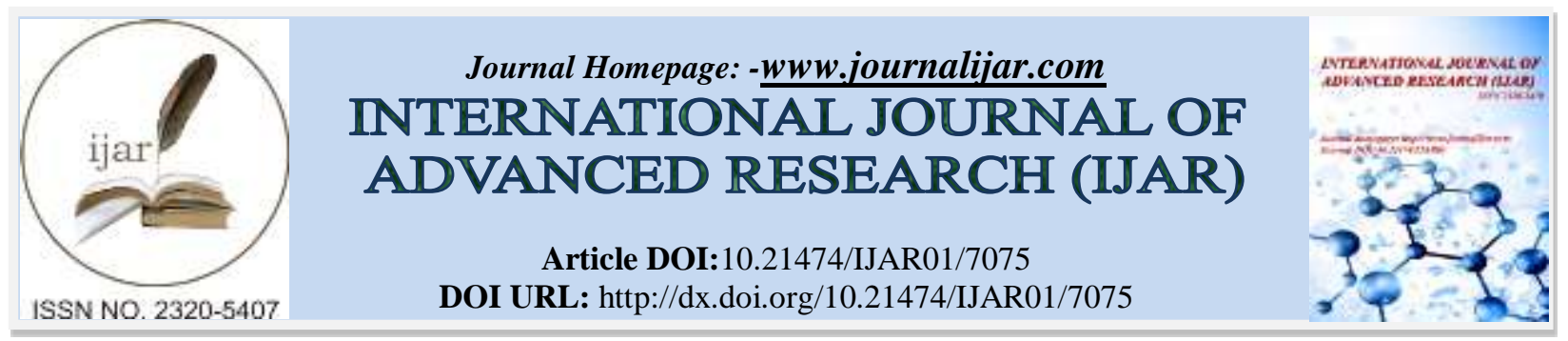

RESEARCH ARTICLE

\title{
THE EFFECTS OF THE OLIVE OIL COMPONENTS ON REDUCING THE CARDIOVASCULAR COMPLICATIONS.
}

Asma Adulkarim.

\section{Manuscript Info}

[..........................

Manuscript History

Received: 11 March 2018

Final Accepted: 13 April 2018

Published: May 2018

\section{Abstract}

Background: Cardiovascular diseases are responsible for more than 7 million deaths worldwide. Cardiovascular risk factors such as hypertension, diabetes and smoking, contribute to the onset of the inflammatory process in atherosclerosis. It has been found that the inclusion of potentially cardioprotective foods, such as olive oil to the diet may aid in the control of the cardio inflammatory markers. Olive oil components, specially the antioxidants such as carotenoids, polyphenolic and -tocopherol compounds (Vit E) have emphasized substantial cardio protective effects. It can also protect against free radicals and lipid peroxidation in humans and prevent the DNA damage.

Objective: The objective of this study is to review the effects of olive oil components on reducing the Cardiovascular complications.

Results: Many Studies demonstrated the association between consumption of extra-virgin olive oil and reduced risk of major cardiovascular events in patients at high risk for developing cardiovascular diseases. In addition, the nonglyceride fraction of Olive Oil is rich in antioxidants such as the carotenoids and polyphenolic compounds. The primary polyphenols are oleuroepein and hydroxytyrosol. Although these substances exist in relatively low concentrations in olive oil, its daily consumption augments the overall antioxidant content in the human body and protects against free radicals and lipid peroxidation in humans.

Conclusion: The role of virgin olive oil components on cardiovascular diseases has been demonstrated by a growing number of studies. However, its exact mechanism remains to be un-specified.

It should be noted that, although Olive Oil influence cardiovascular health, a host of confounding variables, such as lifestyle, exercise, stress level, environment, and genetics, play a role in the individual health.

Copy Right, IJAR, 2018,. All rights reserved.

\section{Introduction:-}

Olive oil is composed mainly of triacylglycerol and contains small quantities of free fatty acids (FFA), glycerol, phosphatides, pigments, flavor compounds, sterols, and microscopic bits of olive (1). 
Coronary artery disease (CAD) is responsible for more than 7 million deaths worldwide. In the early stages of the development of atherosclerotic plaques, cardiovascular risk factors such as hypertension, dyslipidemia, diabetes mellitus, and smoking, contribute to the onset of the inflammatory process in atherosclerosis. The inclusion of potentially cardioprotective foods, such as olive oil, to the diet, may aid in the control of these risk factors, and in the reduction of cytokines and inflammatory markers (8).

Several studies have emphasized the substantial cardio protective effects of olive oil antioxidants such as carotenoids, polyphenolic and -tocopherol compounds (Vit E). In addition the anti oxidanat compounds can also protects against free radicals and lipid peroxidation in humans and prevent the DNA damage.

beside the antioxidant activity, anti-inflammatory effects have been assigned to olive oil phenolic compounds such as OL (oleuropein) and HT(hydroxytyrosol). Thus, nutritional strategies that include olive oil to the daily diet could minimize the inflammatory process in individuals at high cardiovascular risk and help in the primary prevention of CAD (4).

\section{Research Objective:-}

The objective of this study is to review the effects of the olive oil components on reducing the Cardiovascular complications.

\section{Olive Oil Chemicla Componants:- \\ Fatty Acids:-}

The fatty acid composition of olive oil varies widely depending on the cultivar, maturity of the fruit, altitude, climate, and several other factors.

A fatty acid has the general formula: $\mathrm{CH} 3(\mathrm{CH} 2) \mathrm{nCOOH}$ where $\mathrm{n}$ is typically an even number between 12 and 22 (1).

The major fatty acids in olive oil triacylglycerols are:-

1. Oleic Acid (C18:1), a monounsaturated omega- 9 fatty acid. It makes up 55 to $83 \%$ of olive oil.

2. Linoleic Acid (C18:2), a polyunsaturated omega- 6 fatty acid that makes up about 3.5 to $21 \%$ of olive oil.

3. Palmitic Acid (C16:0), a saturated fatty acid that makes up 7.5 to $20 \%$ of olive oil.

4. Stearic Acid (C18:0), a saturated fatty acid that makes up 0.5 to $5 \%$ of olive oil.

5. Linolenic Acid (C18:3) (specifically alpha-Linolenic Acid), a polyunsaturated omega-3 fatty acid that makes up 0 to $1.5 \%$ of olive oil (2).

Triacylglycerols are normally composed of a mixture of three fatty acids. Most prevalent in olive oil is the oleicoleic-oleic (OOO) triacylglycerol, followed, in order of incidence, by palmitic-oleic-oleic (POO), then oleic-oleiclinoleic (OOL), then palmitic-oleic-linoleic (POL), then stearic-oleic-oleic (SOO), and so on (2).

Olive oil contains more oleic acid and less linoleic and linolenic acids than other vegetable oils, that is, more monounsaturated than polyunsaturated fatty acids. This renders olive oil more resistant to oxidation because generally, the greater the number of double bonds in the fatty acid, the more unstable and easily broken down by heat, light, and other factors (2).

In scientific writing the term essential fatty acid refers to all the omega- 3 or omega- 6 fatty acids. This is a historical convention, not a medical definition. From a medical point of view, essential

fatty acids are the ones that the human body cannot make. There are only two, which are the building blocks from which many of the other omega- 3 and omega- 6 fatty acids are made in a healthy body: linoleic acid and alphalinolenic acid (2).

\begin{tabular}{|l|l|}
\hline TABLE 1 : OLIVE OIL FATTY ACIDS & virgin olive oil \\
\hline Saturated fatty acid & $\bullet 7.5$ to $20 \%$ \\
\hline - Palmitic Acid (C16:0) & $\bullet 0.5$ to $5 \%$ \\
\hline - Stearic Acid (C18:0) & $\bullet 12.8 \%$ \\
\hline total saturated & \\
\hline unsaturated fatty acid & $\cdot 3.5$ to $21 \%$ \\
\hline - Linoleic Acid (C18:2) &
\end{tabular}


- Linolenic Acid (C18:3)

- Oleic Acid (C18:1)

- 0 to $1.5 \%$

- 55 to $83 \%$

\section{Polyphenols (Antioxidants):-}

The flavenoid polyphenols in olive oil are natural antioxidants that contribute to a bitter taste, astringency, and resistance to oxidation. They have been shown to have a host of beneficial effects from healing sunburn to lowering cholesterol, blood pressure, and risk of coronary disease (3).

Hydroxytyrosol and tyrosol are some of the many phenol compounds in olive oil (3).

\section{Vitamins:-}

Vitamins can be divided into the fat soluble and water-soluble varieties. Fat soluble vitamins found in olive oil, are generally not broken down by cooking. They are stored in the liver and body fat for long periods so it is not essential to eat them with every meal. Cured whole olives have both water and fat soluble vitaminS.

Vitamin E: (a natural antioxidant): Olives have 1.6mg, or 2.3 IU (International Units) per tablespoon. One tablespoon provides $8 \%$ of RDA for vitamin E (3).

\section{Polycyclic Aromatic Hydrocarbons (Pahs):-}

Many foods naturally contain small quantities of PAHs. Olive oil, like other vegetable cooking oils, has been found to contain minute amounts of up to 17 PAHs such as benzanthracene and chrysene. Unripe olives tended to have more than ripe olives.

Burning any cooking oil can increase the amounts of PAHs. This is not considered a major risk source in the diet and the oil would have to be heated repeatedly and for extended periods to the smoking point. It is unlikely that, in home use, olive oil or other cooking oils would be a significant source of PAHs (3).

\section{Effect of Olive Oil on the Cardiovascular Diseases:-}

The inclusion of potentially cardioprotective foods, such as olive oil, to the diet, may aid in the control of these risk factors, and in the reduction of cytokines and inflammatory markers.

The following Studies demonstrated the association between consumption of extra-virgin olive oil (EVOO), naturally rich in phenolic compounds, and reduced risk of major cardiovascular events in patients at high risk for developing cardiovascular diseases. Such an effect may be mediated by the control of modifiable risk factors and potential anti-inflammatory mechanisms of olive oil phenols (3).

The study conducted by Ros, E.et al. (2006) to address the interaction between phenolic compounds present in extra virigin olive oil (EVOO), and reducing the risk of CAD. In vitro and in vivo studies suggest that phenolic compounds, such as hydroxytyrosol, tyrosol, and their secoiridoid derivatives, may reduce the expression of adhesion molecules and consequent migration of immune cells and decrease circulating levels of inflammatory markers (4).

Another study found that, daily consumption of olive oil seems to modulate cytokines and inflammatory markers related to $\mathrm{CAD}$ in individuals at risk for cardiovascular diseases (8). However, the clinical studies that have evaluated the effects of olive oil and its phenolic compounds on individuals with CAD are still scarce (4).

Studies have shown the anti-inflammatory effects of virgin olive oils supplementation at different stages of development of atherosclerosis, In individuals with endothelial dysfunction, Widmer et al., (2016) evaluated the effects of virgin olive oil (VOO), total polyphenols and enriched with epigallocatechin 3-gallate (EGCG), a catechin naturally found in green tea on inflammatory mediators. The main difference in phenolic composition between the two oils was the secoiridoid content, whereas the lignan content was similar. The authors did not find differences between the VOO and VOO + EGCG groups, but concluded that the supplementation of olive oil to the usual diet of the participants for 4 months had a positive effect on the reduction of cell adhesion molecules (sICAM1), platelets, monocytes, and lymphocytes involved in the inflammatory process (5) 
On the study investigated the Mediterranean diet (MeDiet) supplemented with EVOO (1 liter/week), compared to the control group (low-fat diet) without supplementation their diet with EVOO. They found that the Mediterranean diet (MeDiet) supple0 with EVOO was able to significantly reduce proinflammatory cytokines (proteins that regulate immune and inflammatory responses. (IL6) (P-selectin, sVCAM and sICAM) at short- (3 months) and long-term (1 year) follow-up, in subjects at high cardiovascular risks such as: overweight, obesity, smoking and family history of premature CAD)(6).

Two randomized crossover clinical trials evaluated the anti-inflammatory effects of EVOO (virgin olive oil ), and ROO (refined olive oil )in patients with mild dyslipidemia (an abnormal amount of lipids (e.g. triglycerides, cholesterol and/or fat phospholipids) in the blood). The predominant fatty acids in the two samples were oleic (EVOO vs. ROO), palmitic, and linoleic. The results demonstrated a $20 \%$ reduction in serum TXB2 concentration only in the EVOO group (7).

On the study conducted by Priscilla Azambuja Lopes de Souza, (2017) Obese subjects received a breakfast containing milk and muffins made with different oils:

VOO (virgin olive oil) — containing antioxidant phenols (monounsaturated fatty acid, polyunsaturated fatty acids, saturated fatty acids)

SFO (sun flower oil) - (monounsaturated fatty acid 3, polyunsaturated fatty acids, saturated fatty acids)

SOD, (SFO + canola oil) (artificial antioxidant) (monounsaturated fatty acid, polyunsaturated fatty acids, saturated fatty acids)

SOP (natural antioxidants from olives) - mixture of oils (SFO + canola oil) + VOO-phenol compounds extracted from the residue of olive oil production alperujo (monounsaturated fatty acid, polyunsaturated fatty acid, saturated fatty acids),

They found that, interventions with VOO and SOP reduced NF- $\kappa$ B activation, increased NF- $\kappa$ B alpha inhibitor, and reduced plasma (lipopolysaccharide) LPS concentration. The results suggest that oils rich in phenolic compounds are capable of modulating postprandial inflammation (8).

Several studies have emphasized the importance of a regular use of olive oil in the benefits of traditional mediterranean diet on cardiovascular diseases. In particular, beside the antioxidant activity, vasodilatory, antiplatelet aggregation and anti-inflammatory effects have been assigned to olive oil phenolic compounds such as OL (oleuropein) and HT(hydroxytyrosol) (9).

Several reports have described the protective effects against atherosclerosis of OL and HT in preclinical experimental models.

Visioli et al. (2006) have demonstrated that OL and HT inhibit copper sulphate-induced oxidation of LDL. OL and HT exert a scavenging effect towards $\mathrm{HOCl}$, which acts as an initiating agent in LDL lipid peroxidation, and this effect determines a retard in the onset of the atherosclerotic damage (9). In addition, Jemai et al. (2013) demonstrated that in rats fed with a cholesterol-rich diet, the same compounds were able to promote hypocholesterolemia, lowering LDL plasma levels and total cholesterol; also, they increased the levels of highdensity lipoproteins (HDL) and the activity of antioxidant enzymes reducing LDL oxidation (9).

Recently, the European Food Safety Authority (EFSA) has recognized protective effects of the olive oil phenolic compounds on LDL oxidation, in particular of HT (9).

Carluccio et al. (2013) described the inhibition of endothelial activation, an early step in atherogenesis, by OL and HT, able to reduce lipopolysaccharide (LPS)-stimulated expression of vascular adhesion molecule-1 (VCAM-1) in human vascular endothelial cells by inhibition of its mRNA levels, thus decreasing monocyte cell adhesion to endothelial cells (10).

The two additional mechanisms involved in the vascular damages, which are: platelet aggregation and proliferation of smooth muscle cells, are also antagonized by the olive oil phenolic compounds (10).

The first in vivo experimental study suggested the possibility of using an olive constituent in the treatment of ischemia disease (inadequate blood supply to an organ or part of the body, especially the heart muscles). Rabbits 
were fed diets of refined olive oil, EVOO, and high oleic sunflower oil. The EVOO rabbits demonstrated no change in cholesterol or vitamin E levels after 6 weeks. They had a 30\% longer lag phase before oxidation compared with the refined olive oil and sunflower oil group (11).

Although the Mediterranean diet varies somewhat regionally, its nutritional model of whole grains, vegetables, fruits, and olive oil is believed to contribute to decreased rates of coronary heart disease. A recent survey in the region of Girona, Spain, of 3,179 sub-jects found that adherence to the traditional Mediterranean diet was inversely associated with body mass index and obesity, which are the major risk factors for heart disease (12).

The low incidence of cardiovascular disease in countries bordering the Mediterranean basin, where olive oil is the main source of dietary fat, and the negative association between this disease with high density lipoproteins has stimulated interest. Cumulative evidence suggests that high density lipoprotein (HDL) cholesterol, and its main apolipoprotein A1, may be increased by consuming olive oil when compared with carbohydrate and low fat diets in humans (18).

Many studies affirm that dietary cis-Monounsaturated fatty acids (MUFA) have a greater anti-atherosclerotic effect than saturated fatty acids (SFA) and are comparable with the effects of polyunsaturated fatty acids (PUFA) on cardiovascular risk factors (13).

It should be noted also, that although diet does influence cardiovascular health, a host of confounding variables, such as lifestyle, exercise, stress level, environment, and genetics, play a role in the health of those in the Mediterranean region.

In addition to the substantial effects of olive oil on the vascular and its cardio protective effects. The nonglyceride fraction of EVOO is rich in antioxidants such as the carotenoids and polyphenolic compounds. The primary polyphenols are oleuroepein, hydroxytyrosol, and -tocopherol. Hydroxytyrosol is a byproduct of oleu- ropeintocopherol, also known as an active form of vitamin E, is highly resistant to oxidative degradation. Although it exists in relatively low concentrations in olive oil, its daily consumption augments the overall antioxidant content in the human body and protects against free radicals and lipid peroxidation in humans (16).

Polyphenols interfere with the chain of reactions initiated and supported by free radicals.

This prevents DNA damage, lipid hydroperoxide formation, and lipid peroxidation.

Conflicting results have been found in many studies when olive oil diets were compared with other sources of fat. The role of virgin olive oil minor components on its protective effect has been demonstrated by a growing number of studies although its exact mechanism remains to be elucidated (18). Further studies in this field in humans and in animal models are warranted

Hydroxytyrosol is one of the major phenolic compounds in olive oil and has demonstrated strong radical-scavenging properties. Several studies have been performed in order to look further into the effects of the polyphenol hydroxytyrosol in relation to cardiovascular events and illnesses in animal trials and in vitro. However, no clinical trials have focused on the specific action of hydroxytyrosol and cardiovascular diseases, although some are being undertaken to look at olive oil or olive leaf extract properties (19).

As we stated earlier, olive oil phenolics have positive effects on certain physiological parameters, such as plasma lipoproteins, oxidative damage, inflammatory markers, platelet, cellular function, and antimicrobial activity. However, number of factors could affect phenolic concentrations in virgin olive oil, so it is of great importance to understand these factors in order to preserve the essential health promoting benefits of olive oil phenolic compounds (21)

\section{Conclusion:-}

1. In the Cardiovascular diseases including the early stages of the development of atherosclerotic plaques and Coronary artery disease, the inclusion of potentially cardio protective foods, such as olive oil to the diet may aid in the control of cardiovascular risk factors such as hypertension, dyslipidemia, diabetes and smoking.In addition to the substantial effects of olive oil on the vascular and its cardio protective effects. 
2. The nonglyceride fraction of Extra Virgin Olive Oil (EVOO) is rich in antioxidants such as the carotenoids and polyphenolic and -tocopherol compounds (Vit. E), which has substantial cardio protective effects. In addition, the antioxidant compounds can also protect against free radicals and lipid peroxidation in humans. This also prevents DNA damage, lipid hydroperoxide formation.

3. It should be noted that, although Olive Oil influence cardiovascular health, a host of confounding variables, such as lifestyle, exercise, stress level, environment, and genetics, play a role in the individual health.

4. The role of virgin olive oil components on cardiovascular diseases has been demonstrated by a growing number of studies. However, its exact mechanism remains to be un-specified. More research needs to be conducted especially at the cellular level, to more fully understand the pathways by which olive oil help to reduce cardiovascular disease risk factors.

\section{References:-}

1. Paul, K. Apostolos K. Elizabeth B. Walter C. Willet, Ruben J. Hernandez Olive Oil, From the Tree to the Table, Chronicle Books, (1997)

2. Tous, J. and L. Ferguson. Mediterranean fruits. p. 416-430. In: J. Janick (ed.), Progress in new crops. ASHS Press, Arlington, VA.( 1996.)

3. Goulart; Sawaya et al.; Fernandez Diez; IBPGR; Morton; Cantwell (1994).

4. Estruch, R.; Ros, E. et al. Primary prevention of cardiovascular disease with a Mediterranean diet.

5. Santangelo, C.; Filesi, C.; Varì, R.; Scazzocchio, B.; Filardi, T.; Fogliano, V.; D’Archivio, M.; Giovannini, C.; Lenzi, A.; Morano, S.; et al. Consumption of extra-virgin olive oil rich in phenolic compounds improves metabolic control in patients with type 2 diabetes mellitus: A possible involvement of reduced levels of circulating visfatin. J. Endocrinol. Investig. (2016), 39, 1295-1301.

6. Casas, R.; Urpi-Sardà, M.; Sacanella, E.; Arranz, S.; Corella, D.; Castañer, O.; Lamuela-Raventós, R.M.; SalasSalvadó, J.; Lapetra, J.; Portillo, M.P.; et al. Anti-Inflammatory Effects of the Mediterranean Diet in the Early and Late Stages of Atheroma Plaque Development. Med. Inflamm. (2017), 1-12

7. Moreno-Luna, R.; Muñoz-Hernandez, R.; Miranda, M.L.; Costa, A.F.; Jimenez-Jimenez, L.; Vallejo-Vaz, A.J.; Muriana, F.J.; Villar, J.; Stiefel, P. Olive oil polyphenols decrease blood pressure and improve endothelial function in young women with mild hypertension. Am. J. Hypertens( 2012), 25, 1299-1304.

8. Priscilla Azambuja Lopes de Souza, Aline Marcadenti, and Vera Lúcia Portal (2017 ).

9. Estruch R1, Ros E, Salas-Salvadó J, Covas MI, Corella D, Arós F, Gómez-Gracia E, Ruiz-Gutiérrez V, Fiol M, Lapetra J, Lamuela-Raventos RM, Serra-Majem L, Pintó X, Basora J, Muñoz MA, Sorlí JV, Martínez JA, Martínez-González MA; PREDIMED Study Investigators .Epub (2013 Feb 25.)

10. Bulotta et al.; licensee BioMed Central Ltd. 10.1186/s12967-014-0219-9

11. Ioanna Andreadou, Efstathios K. Iliodromitis, Emmanuel Mikros, Maria Constantinou, Apostolos Agalias, Prokopios Magiatis, Alexios Leandros Skaltsounis, Elli Kamber, Anna Tsantili-Kakoulidou, and Dimitrios Th Kremastinos

12. Schroder H, Marrugat J, Vila J, Covas M. Adherence to the traditional Mediterranean diet is inversely associated with body mass index and obesity in a Spanish population. J Nutr (2004); 134:3355-3361.

13. Kris-EthertonP.AHAScienceAdvisory:monounsaturatedfatty acids and risk of cardiovascular disease. J Nutr (1999);129:2280- 2284

14. Boskou D. Olive oil: chemistry and technology. Champaign, IL: AOCS Press. Received December 14, 2007; Revised January 31, 2008; Accepted February 11, 2008.ISSN 1072-7515/08

15. Perona J, Cabello-Moruno R, Ruiz-Guiterrez V. The role of virgin olive oil components in the modulation of endothelial function. J Nutr Biochem 2006;17:429-445.

16. Perona J, Cabello-Moruno R, Ruiz-Guiterrez. J Nutr Biochem 2006;17:429-445.

17. Jose M. Lou-Bonafonte, Montse Fito, Maria-Isabel Covas, Marta Farras, Jesus Osada. 10.2174/157016112800812827

18. Silvia Tejada, Samuel Pinya, Maria del Mar Bibiloni, Josep A. Tur, Antoni Pons, Antoni Sureda*.10.2174/1389450117666161005150650

19. Guillén N, Acín S, Navarro MA, Surra JC, Arnal C, Lou-Bonafonte JM, Muniesa P, Martínez-Gracia MV, Osada J. 10.1016/S1885-5857(09)71560-9

20. Cicerale S, Conlan XA, Sinclair AJ, Keast RS.10.1080/10408390701856223.

21. Ros E, Martínez-González MA, Estruch R, Salas-Salvadó J, Fitó M, Martínez JA, Corella D. 10.3945/an.113.005389. 